\title{
Lobular carcinoma in situ of the breast - correlation between minimally invasive biopsy and final pathology
}

\author{
Bartłomiej Szynglarewicz¹, Piotr Kasprzak², Agnieszka Hałoń ${ }^{3}$, Rafał Matkowski11,4
}

${ }^{1}$ Department of Surgical Oncology, Lower Silesian Oncology Centre, Wroclaw, Poland ${ }^{2}$ Department of Breast Imaging, Lower Silesian Oncology Centre, Wroclaw, Poland ${ }^{3}$ Department of Pathology, Wroclaw Medical University, Wroclaw, Poland

${ }^{4}$ Chair of Oncology, Wroclaw Medical University, Wroclaw, Poland

Submitted: 4 May 2015

Accepted: 30 June 2015

Arch Med Sci 2017; 13, 3: 617-623

DOI: 10.5114/aoms.2016.61815

Copyright $\odot 2016$ Termedia \& Banach

\section{Abstract}

Introduction: Lobular carcinoma in situ (LCIS) is regarded as a non-obligate precursor of invasive breast cancer (IBC). Hence, the optimal management of LCIS found on minimally invasive breast biopsy remains a subject of debate. The aim of this study was to evaluate the correlation of biopsy findings with postoperative histology and to identify risk factors for upstaging to IBC. Material and methods: Twenty-seven patients with pure LCIS diagnosed on image-guided biopsy (vacuum-assisted or core-needle) underwent subsequent surgery. Clinical, radiological and histological features were compared to the final pathology after surgical excision.

Results: Median age of patients was 56 years while median size of LCIS was $15 \mathrm{~mm}$. Final examination demonstrated IBC foci in $29.6 \%$ of lesions. Upstaged patients were younger and had larger lesions but without statistical significance ( $p=0.07$ and $p=0.09$, respectively). Palpable tumours $(p=$ $0.0004)$, BIRADS 5 lesions $(p=0.0001)$, masses $(p=0.016)$ and pleomorphic LCIS $(p=0.0001)$ had a significantly increased rate of upstaging. Guidance of the procedure (ultrasound vs. stereotactic) was significantly associated with the upstaging risk $(p=0.016)$, while the importance of the biopsy technique (core-needle vs. vacuum-assisted) was not confirmed $(p=0.37)$. After excluding pleomorphic LCIS and mass-forming classic LCIS, there was no risk of upstaging for lesions with BIRADS 4 mammographic abnormalities.

Conclusions: Pleomorphic histology, mass formation and BIRADS 5 category reflect more aggressive behaviour of LCIS and identify patients who need subsequent surgery. For other patients, close follow-up could be a safe alternative.

Key words: breast cancer, lobular carcinoma in situ, core-needle biopsy, vacuum-assisted biopsy.

\section{Introduction}

Although systemic therapy of breast cancer is more and more effective and targeted, surgery remains the gold standard of treatment in most cases $[1,2]$. However, regarding borderline lesions found on minimally invasive biopsy the need of subsequent surgical excision is still under investigation. In the era of widespread screening programmes, preoperative diagnosis of minimal breast neoplasia is more and more common. In addition, a considerable increase in the incidence of lobular pre-invasive disease in recent decades has been observed [3].

\author{
Corresponding author: \\ Bartłomiej Szynglarewicz \\ $\mathrm{MD}, \mathrm{PhD}$ \\ Department \\ of Surgical Oncology \\ Lower Silesian \\ Oncology Centre \\ pl. Hirszfelda 12 \\ 53-413 Wroclaw, Poland \\ Phone: +48 664429462 \\ E-mail: szynglarewicz.b@dco. \\ com.pl
}


The entire spectrum of lobular proliferation, including atypical hyperplasia (ALH) and carcinoma in situ (LCIS), is covered by the term lobular neoplasia (LN) [4] or, more recently, by the term lobular intraepithelial neoplasia (LIN) [5]. These terms reflect the heterogenic malignant potential of these lesions and are less anxiety-producing than the name carcinoma. However, the distinction between ALH and LCIS is postulated by some because of the substantial difference in the risk of subsequent invasive cancer, associated with the extent of lobular proliferation [6]. There is still no consensus whether LCIS is just a marker of an increased risk of invasive malignancy (ipsi- and contralateral, lobular and ductal) or represents a direct precursor of invasive breast cancer (IBC) $[7,8]$. Hence, the optimal management of LCIS found on minimally invasive breast biopsy remains a subject of debate.

The aim of this study was to evaluate the correlation of biopsy findings with postoperative histology and to identify risk factors for upstaging to IBC.

\section{Material and methods}

Twenty-seven women with LCIS diagnosed from 2004 to 2014 were studied. All of them underwent percutaneous breast biopsy (stereotactic or ultrasound-guided) of a lesion suspicious of malignancy (BIRADS 4) or highly suggested for cancer (BIRADS 5). In patients with masses ultrasound-guided biopsy was carried out: 14-gauge core needle biopsy with an automated gun (Manan Pro-Mag 2.2, Angiotech Pharmaceuticals Inc, distributed in Europe by PBN Medicals, Stenlose, Denmark) for tumours larger than $20 \mathrm{~mm}$ and vacuum-assisted biopsy for smaller masses using an 11- or 10-gauge needle (Mammotome System MHH 11, Ethicon EndoSurgery Europe, Norderstedt, Germany; and EnCore Enspire Breast Biopsy System, C.R. Bard Inc., Tempe, AZ, USA, respectively). In patients with other lesions, not well seen in ultrasound, a stereotactic biopsy under digital mammography guidance was completed using a designated prone table unit with the 11-G Mammotome device MST 11 (Mammotest Plus/S, Fisher Imaging, Denver, USA). Biopsies were performed strictly according to the standardised protocol of this procedure, with 5 specimens as a minimal number of tissue cores.

In all the cases histological examination reported pure LCIS (classic or pleomorphic) without any invasive component. In cases with any doubt about the origin, E-cadherin immunochemistry was used. Subsequently, patients underwent surgical excision after image-guided hook-wire or skin marking localisation. Postoperative pathologic assessment of the surgical specimen was performed, with a special effort to identify any foci of $I B C$ in residual disease. Biopsy findings were compared to the final pathology.

\section{Statistical analysis}

Medians, means, standard deviations and ranges were calculated when appropriate. With regard to the potential risk factors, rates of upstaging to IBC were calculated. Continuous variables were compared between 2 groups using the Mann-Whitney U-test or Student's $t$-test, when appropriate. Categorical variables were analysed using Pearson's $\chi^{2}$ test. $P$-values less than 0.05 were considered statistically significant.

\section{Results}

Median age of patients (mean \pm SD, range) was 56 years (56.6 $\pm 9.7,36-74$ ), while median size of LCIS was $15 \mathrm{~mm}(15.3 \pm 5.9,6-28)$. Seven core-needle and 20 vacuum-assisted biopsies were done, 13 under mammography and 14 under ultrasound guidance. Ten palpable and 17 nonpalpable lesions were investigated, 18 categorised as BIRADS 4 and 9 as BIRADS 5. Characteristics of patients, lesions and procedures referring to all the patients and separately to upstaged and not upstaged groups are given in Table I.

Final pathology of surgical specimen demonstrated IBC foci in $29.6 \%$ of lesions (8/27): 3 cases of lobular IBC, 3 of ductal IBC, and 2 of ductal IBC with an intraductal component (ductal carcinoma in situ - DCIS). Patient age did not differ significantly between upstaged and not upstaged groups (median, mean \pm SD): 48.5, $50.9 \pm 13.0$ vs. $56,59.1 \pm 7.1$ years, respectively. However, upstaged patients tended to be distinctively younger, close to statistical significance ( $p=0.07, U$-value: 42, Z-ratio: 1.78). Not upstaged lesions were smaller (median, mean \pm SD): 14, $13.8 \pm 5.2$ vs. $17,18.6 \pm 6.6 \mathrm{~mm}$, respectively. Again, the difference did not reach statistical significance, but was near ( $p=0.09, U$-value: 44.5, Z-ratio: 1.64 ). When considering the diameter of 10,15 and $20 \mathrm{~mm}$ as cut-off points, lesion size was not important in the $\chi^{2}$ test $(p=0.43, p=0.33$ and $p=0.37$, respectively). Features significantly related to the increased risk of upstaging were: palpability, BIRADS category 5 , presentation as a mass, ultrasound-guided biopsy and pleomorphic type of LCIS ( $p=0.0004$, $p=0.0001, p=0.0161, p=0.016$ and $p=0.0001$, respectively). Biopsy technique (core-needle vs. vacuum-assisted) showed a lack of importance $(p=0.37)$. Details are presented in Table II.

All five pleomorphic LCIS were upstaged. All of them presented as BIRADS 5 hypoechoic, irregular masses with posterior shadowing and underwent biopsy under ultrasound guidance. Among three upstaged classic type LCIS there were two 
Table I. Comparison between not-upstaged and upstaged group

\begin{tabular}{|lccc|}
\hline Features & $\begin{array}{c}\text { All LCIS patients } \\
(n=27)\end{array}$ & $\begin{array}{c}\text { Not upstaged } \\
(n=19)\end{array}$ & $\begin{array}{c}\text { Upstaged } \\
(n=8)\end{array}$ \\
\hline $\begin{array}{l}\text { Patient age: } \\
\text { Median, mean } \pm \text { SD, range }\end{array}$ & $56,56.6 \pm 9.7,36-74$ & $56,59.1 \pm 7.1,47-74$ & $48.5,50.9 \pm 13.0,36-71$ \\
\hline $\begin{array}{l}\text { LCIS size [mm]: } \\
\text { Median, mean } \pm \text { SD, range }\end{array}$ & $15,15.3 \pm 5.9,6-28$ & $14,13.8 \pm 5.2,6-22$ & $17,18.6 \pm 6.6,9-28$ \\
\hline $\begin{array}{l}\text { Radiological presentation: } \\
\text { calcifications/AD/FAD/mass }\end{array}$ & $11 / 1 / 1 / 14$ & $10 / 1 / 1 / 7$ & $1 / 0 / 0 / 7$ \\
\hline $\begin{array}{l}\text { Radiological category: } \\
\text { BIRADS 4/BIRADS 5 }\end{array}$ & $18 / 9$ & $17 / 2$ & $1 / 7$ \\
\hline $\begin{array}{l}\text { Clinical character: } \\
\text { Palpable/non-palpable }\end{array}$ & $10 / 17$ & $3 / 16$ & $7 / 1$ \\
\hline $\begin{array}{l}\text { Biopsy guidance: } \\
\text { Stereotactic/ultrasound }\end{array}$ & $13 / 14$ & $12 / 7$ & $1 / 7$ \\
\hline $\begin{array}{l}\text { Biopsy technique: } \\
\text { Core-needle/vacuum-assisted }\end{array}$ & $7 / 20$ & $4 / 15$ & $3 / 5$ \\
\hline $\begin{array}{l}\text { Histological type: } \\
\text { Classic/pleomorphic }\end{array}$ & $22 / 5$ & $19 / 0$ & $3 / 5$ \\
\hline
\end{tabular}

$A D$ - architectural distortion, FAD - focal asymmetric density.

masses (BIRADS 5 and BIRADS 4) and one lesion radiologically detected as BIRADS 5 microcalcifications. Mass presentation and BIRADS category 5 were significantly related to pleomorphic histology when compared to classic type $(p=0.017$ and $p=0.0004$, respectively). Pleomorphic type was associated with larger lesion size with statistical significance (median, mean $\pm \mathrm{SD}$, range): 22, $20.2 \pm 8.9,9-28$ vs. $14.5,14.1 \pm 4.9,6-22 \mathrm{~mm}$ ( $p=0.037$, $t$-value: 2.194$)$. After excluding pleomorphic LCIS and mass-forming classic LCIS, there was no risk of upstaging for lesions with BIRADS 4 mammographic abnormalities.

\section{Discussion}

Since the LCIS is regarded as a non-obligate precursor of IBC, the need of surgical excision when it is diagnosed on minimally invasive breast biopsy remains an open question [8]. Some still recommend LCIS to be managed with subsequent surgery as a highest risk lesion [9]. It is based on the significant risk of upstaging to IBC on final pathology of the surgical specimen, being even as high as $40-50 \%$ in some series [10-12]. However, the upgrading rates reported during recent decade have varied remarkably. In addition, because LCIS is a relatively rare disease, most studies are retrospective, observational, and of small sample size. No wonder that the findings are discrepant and any unequivocal conclusions are hard to draw.

Renshaw et al. studied prospectively a large cohort of 467 women with LN on core biopsy. Among 92 patients with pure LN and available follow-up data just 3 cancers (2 IBC and 1 DCIS) were found in surgical excision of the biopsy site, giving a $3.3 \%$ rate of upgrading [13]. The
Table II. Risk factors for upstaging LCIS to invasive cancer

\begin{tabular}{|c|c|c|}
\hline Variables & Rate of upstaging & $P$-value \\
\hline \multicolumn{3}{|c|}{ Radiological category: } \\
\hline BIRADS 5 & $77.8 \%(7 / 9)$ & $<0.001$ \\
\hline BIRADS 4 & $5.6 \%(1 / 18)$ & \\
\hline \multicolumn{3}{|c|}{ Radiological presentation: } \\
\hline Mass & $50 \%(7 / 14)$ & $<0.05$ \\
\hline Other & $7.7 \%(1 / 13)$ & \\
\hline \multicolumn{3}{|l|}{ Clinical character: } \\
\hline Palpable & $70 \%(7 / 10)$ & $<0.001$ \\
\hline Non-palpable & $5.9 \%(1 / 17)$ & \\
\hline \multicolumn{3}{|l|}{ Biopsy technique: } \\
\hline Core-needle & $42.9 \%(3 / 7)$ & 0.373 \\
\hline Vacuum-assisted & $25 \%(5 / 20)$ & \\
\hline \multicolumn{3}{|l|}{ Biopsy guidance: } \\
\hline Ultrasound & $50 \%(7 / 14)$ & $<0.05$ \\
\hline Stereotactic & $7.7 \%(1 / 13)$ & \\
\hline \multicolumn{3}{|l|}{ Histological type: } \\
\hline Pleomorphic & $100 \%(5 / 5)$ & $<0.001$ \\
\hline Classic & $13.6 \%(3 / 22)$ & \\
\hline
\end{tabular}

authors compared their results to findings from other reports. This multi-institutional analysis of 12 series revealed considerable higher risk of upstaging. Among 190 women with core-diagnosed LN who underwent subsequent opera- 
tion 23 (12.1\%) had IBC and 16 (8.4\%) had DCIS which gives an upstaging rate of over $20 \%$ [13]. This contrast demonstrates how much the significance of LN on core needle biopsy specimens differs at different centres. As yet, there is no consensus regarding the optimal management of LCIS, and controversy exists when subsequent surgical excision is needed and in which cases a more selective approach is justified. The upgrading incidence, rates and risk factors in 14 series (including the current study) are presented in Table III.

Mammographic calcifications have not been traditionally considered a typical presentation of LCIS, which can lack any radiographic abnormalities [14]. It is due to the fact that asymptomatic LCIS is usually an unexpected finding on biopsy performed for another lesion (e.g. columnar cell alteration, often associated with LCIS) containing the targeted microcalcifications [15]. However, more recent studies have shown that lobular lesions, particularly LCIS, may contain mammographically evident calcifications [16]. These findings are reinforced by the current literature. Scoggins and colleagues found that $90 \%$ of lesions yielding LCIS had mammographic abnor- malities, most frequently (80\%) grouped amorphous calcifications [17]. Other investigators reported similar association between LCIS and the presence of microcalcifications, which were observed in $75-82 \%$ of cases $[10,11,18]$.

A radiographic mass is generally an unusual presentation of LCIS, substantially less common than other mammographic findings, such as calcifications, architectural distortions, focal asymmetric densities, and stellate lesions without a mass $[10,17]$. Some suggest that due to this rarity a diagnosis of lobular lesion does not provide sufficient explanation for a mammographic or ultrasound mass. As a consequence, in the aspect of imaging-histologic correlation it should be considered a discordant result, therefore warranting surgical intervention [19]. Indeed, mass-forming LCIS was reported to be associated with significantly higher risk of IBC at subsequent surgical excision when compared to the other mammographic targets, particularly microcalcifications [10, 20]. In the present study we observed a statistically significant 6.5 -fold increase of the upstaging rate when the LCIS presented as a radiographic mass. In our previous series invasive lobular cancer was postoperatively found in $64 \%$ of LN with

Table III. Results of follow-up surgical excision of LN diagnosed on core-needle biopsy: literature review and current study

\begin{tabular}{|c|c|c|c|c|c|c|c|}
\hline Study & Ref. & $N$ & ALH & LCIS & LN & $\begin{array}{c}\text { No. of } \\
\text { upgrades (\%) }\end{array}$ & Risk factors for upgrading \\
\hline Middleton (2003) & [22] & 17 & 6 & 9 & 2 & $6(35.3)$ & Mass lesion \\
\hline Arpino (2004) & [16] & 21 & NA & NA & 21 & $3(14.3)$ & - \\
\hline $\begin{array}{l}\text { Elsheikh \& Silverman } \\
(2005)\end{array}$ & [32] & 33 & 20 & 13 & NA & $9(27.3)$ & $\begin{array}{c}\text { Mass lesion, pleomorphic } \\
\text { histology }\end{array}$ \\
\hline Renshaw (2006) & [13] & 92 & 40 & 52 & NA & $3(3.26)$ & - \\
\hline Esserman (2007) & {$[18]$} & 26 & NA & NA & 26 & $2(7.69)$ & Diffuse LCIS $^{b}$ \\
\hline Ciangiarella (2008) & {$[20]$} & 38 & 18 & 20 & NA & $3(7.89)$ & Mass lesion \\
\hline Hwang (2008) & [36] & 87 & 48 & 39 & NA & $10(11.5)$ & $\begin{array}{l}\text { Imaging-histologic discordance, } \\
\text { pleomorphic histology }\end{array}$ \\
\hline Menon (2008) & {$[34]$} & 25 & NA & NA & $25^{a}$ & $9(36.0)$ & $\begin{array}{l}\text { Mass lesion, imaging-histologic } \\
\text { discordance }\end{array}$ \\
\hline Nagi (2008) & [35] & 45 & NA & NA & 45 & $2(4.44)$ & - \\
\hline Polom (2009) & [10] & 20 & 11 & 9 & NA & $8(40.0)$ & $\begin{array}{l}\text { Mass lesion, architectural } \\
\text { distortion on mammography }\end{array}$ \\
\hline Flegg (2010) & [11] & 9 & 4 & 5 & NA & $2(22.2)$ & - \\
\hline Rendi (2012) & {$[24]$} & 68 & 20 & 48 & NA & $3(4.41)$ & $\begin{array}{c}\text { Imaging-histologic discordance, } \\
\text { women at high risk, extensive } \\
\text { LCISc }\end{array}$ \\
\hline Murray (2013) & [19] & 80 & 34 & $46^{\mathrm{a}}$ & NA & $5(6.25)$ & Imaging-histologic discordance \\
\hline Current study & & 27 & NA & 27 & NA & $8(29.6)$ & $\begin{array}{l}\text { Mass lesion, BIRADS category } 5 \text {, } \\
\text { pleomorphic histology, palpability }\end{array}$ \\
\hline Total & & 588 & 201 & 268 & 119 & 73 (12.4) & - \\
\hline
\end{tabular}

NA - not applicable; ${ }^{a}$ classic type, ${ }^{b}>1$ lobule per core; ${ }^{c}>4$ foci. 
radiological symptoms produced by itself and in none of asymptomatic incidental LN. With regard to imaging symptoms, all mass lesions were upstaged, while none with microcalcifications were upstaged. Among upstaged LN, just one lesion did not form a mass but an architectural distortion [21]. Middleton et al. reviewed the experience of MD Anderson Cancer Center and reported that carcinoma was present in $35 \%$ of excised LN. All these lesions were detected as a mass on imaging, and there were no upgrades when microcalcifications were the biopsy target [22]. Similar results were published by Murray et al. from Memorial Sloan-Kettering Cancer Center. In their study onethird of mass-forming LN werer upstaged to IBC or DCIS at surgical excision [19].

Although mammography is the most sensitive method for imaging of radiologically symptomatic $L N$, some of these lesions can be diagnosed on magnetic resonance imaging (MRI), which is often used in surveillance strategies in women at high risk [23]. Rendi et al. investigated 93 cases of LN and reported that $24 \%$ of them were detected by MRI. Pathological non-mass enhancement occurring in $16 \%$ of cases was the second most common finding, just after microcalcifications [24]. In the study of Murray et al., $21 \%$ of LN lesions had an abnormality on contrast-enhanced MRI [19]. In the series of Scoggins et al. $71 \%$ of LCIS detected by MRI were associated with heterogeneous nonmass enhancement [17]. An interesting issue is the utility of MRI examination following excision of $L N$, as these patients have an increased risk of IBC. However, the benefit of MRI in this women cohort is unclear. Friedlander et al. found that MRI identified otherwise occult IBC in $3.8 \%$ of LCIS patients [25], while Sung et al. reported that MRI detected occult cancers in $4.5 \%$ of women with a history of LCIS [26]. In contrast, the studies of Port et al. and King et al. demonstrated that adjunctive MRI screening after diagnosis of LCIS did not translate into a clinical benefit for these women, in terms of an increased cancer detection rate or earlier stage at diagnosis $[27,28]$. The authors explained that although the lifetime IBC risk in women with LCIS may exceed $20 \%$ (depending on age at diagnosis), the biology of these cancers differs from that in women at high risk on the basis of genetic predisposition or strong family history [28]. Moreover, the enhanced surveillance strategy that includes $M R I$ may result in remarkably increased numbers of benign biopsies when compared to conventional screening [29].

Pleomorphic histology LCIS had very strong positive predictive value for invasion in our series; in all these cases foci of IBC were found postoperatively. This histologic form was significantly related to other risk factors: BIRADS category 5, mass presentation, and larger lesion size. Pleomorphic
LCIS is characterised by nuclear pleomorphism, prominent nucleoli, nuclear membrane irregularity, mitotic figures and central necrosis with associated calcifications [7]. Cytologic and histologic findings can make it difficult to distinguish from high-grade ductal carcinoma in situ, but as for other lobular proliferations, E-cadherin expression is usually absent [30]. As opposed to classic form, pleomorphic LCIS is often symptomatic on imaging, has lower ER and PR expression levels, a higher average Ki-67 proliferation index, and is more likely to show HER2/neu overexpression [31]. It is believed to be a more aggressive subtype of LCIS. This concept is supported by clinical studies, including our findings. Elsheikh and Silverman reported that pleomorphic histology was related to significantly increased risk of upstaging after surgical excision [32]. Meroni et al. found the upstaging rate as high as $50 \%$ for pleomorphic LCIS, while it was $12 \%$ and $7 \%$ for classic LCIS and ALH, respectively [12].

Our findings support individual management of LN found on minimally invasive biopsy, warranting surgical excision if $L N$ presents as mass lesion and is of pleomorphic type. Other factors influencing high risk of upstaging have also been identified. Subsequent surgery can be justified in women at high risk of breast cancer [24], when a residual lesion is seen [12], LN is associated with higher BIRADS category and imaging-histologic discordance [19, 33], numerous lobules are involved [18], another high-risk or borderline lesion is present [34], it is not possible to exclude DCIS despite E-cadherin immunochemistry [34], and when necrosis, ductal involvement or atypical ductal hyperplasia is found $[16,22,34]$. This selective approach to surgical excision, based on histology and imaging findings, is also strongly recommended by other investigators [13, 20, 35, 36].

If the surgical excision can be omitted, the question is how safe is a close follow-up as an alternative. Traditionally, it can be considered when the risk of upstaging is not higher than the routinely used $2 \%$ threshold [37]. In our series, after excluding pleomorphic LCIS and mass-forming classic LCIS, there was no risk of upstaging for lesions with BIRADS 4 mammographic abnormalities. In the study of Meroni et al., 11 of 148 $\mathrm{LN}$ patients included in the survival analysis with mean 5.2-year follow-up developed an ipsilateral tumour. The authors suggest that patients with ALH and classic LCIS in vacuum-assisted breast biopsy can be safely monitored by clinical and radiological examinations [12]. Murray et al.reported a $3 \%$ rate of upstaging (2/72) when classic LCIS with imaging-histologic concordance was considered, just above the $2 \%$ threshold. The authors claim that the number of cancers that would have been missed if there had been no excision was 
extremely small and consisted mostly of $2 \mathrm{~mm}$ low-grade DCIS. The authors claim that these microscopic carcinomas, even if undetected, would likely have very minimal, if any, impact on patient survival, especially in patients with $\mathrm{LN}$ who are subject to close follow-up [19]. Similar conclusions can be drawn from the studies of Hwang et al., who observed a $1 \%$ upstaging rate $(1 / 84 ; 4 \mathrm{~mm}$ DCIS) after excluding LCIS with imaging-histologic discordance and non-classic morphology [36], and Rendi et al., who found a $3 \%$ rate (2/67; low-grade DCIS) for imaging-histologic concordant cases [24]. In the series of Parkin et al., just 1 of 11 patients who underwent surgery due to non-pleomorphic LN diagnosed on vacuum-assisted biopsy was upgraded (who had undergone low tissue yield biopsy). In 40 other patients who had biopsy with complete imaging-histologic concordance there were no breast cancers during a mean annual mammographic follow-up period of 53 months. In the authors' opinion, in the management of non-pleomorphic LN vacuum-assisted breast biopsy is a safe option [38].

This report has several important limitations. First, the small number of patients hinders the ability to prove statistical significance. Second, this is a single institution series. The characteristics of patient populations managed in other institutions may be different. Thus, the repeatability of our findings may be limited; hence the results of this study may not be applicable to other specific settings. Third, a variety of minimally invasive biopsy techniques (vacuum-assisted and core-needle with automated gun), image guiding methods (stereotactic and ultrasound-guided), and needle gauges were used. The use of one biopsy technique, image guiding method and needle gauge would have been ideal for the confidence of results.

In conclusion, our results, taken together with findings from recent studies, support the more conservative approach to subsequent surgery when LCIS is found on preoperative breast biopsy. Management should be individually tailored, warranting surgical excision in cases with highrisk factors. In our series, pleomorphic histology, mass formation and BIRADS category 5 identified patients who needed subsequent intervention. In cases with the presence of microcalcifications without a mass, BIRADS category 4 , and classic type of LCIS, the risk of upstaging was very low. Our findings suggest that for women with nonmass-forming classic LCIS without residual disease or imaging-histologic discordance, a close radiological and clinical follow-up can be offered as an alternative to surgery.

\section{Conflict of interest}

The authors declare no conflict of interest.

\section{References}

1. Kołacińska A, Chałubińska J, Błasińska-Morawiec M, et al. Pathological complete response in younger and older breast cancer patients. Arch Med Sci 2012; 8: 310-5.

2. Badowska-Kozakiewicz AM, Sobol M, Patera J, Kozłowski W. Immunohistochemical evaluation of human epidermal growth factor receptor 2 and estrogen and progesterone receptors in invasive breast cancer in women. Arch Med Sci 2013; 9: 466-71.

3. Li Cl, Anderson BO, Daling JR, Moe RE. Changing incidence of lobular carcinoma in situ of the breast. Breast Cancer Res Treat 2002; 75: 259-68.

4. Haagensen CD, Lane N, Lattes R, Bodian C. Lobular neoplasia (so-called lobular carcinoma in situ) of the breast. Cancer 1978; 42: 737-69.

5. Bratthauer GL, Tavassoli FA. Lobular intraepithelial neoplasia: previously unexplored aspects assessed in 775 cases and their clinical implications. Virchows Arch 2002; 440: 134-8.

6. Page DL, Kidd TE Jr, Dupont WD, Simpson JF, Rogers LW. Lobular neoplasia of the breast: higher risk for subsequent invasive cancer predicted by more extensive disease. Hum Pathol 1991; 22: 1232-9.

7. Jorns J, Sabel MS, Pang JC. Lobular neoplasia: morphology and management. Arch Pathol Lab Med 2014; 138: 1344-9.

8. Simpson PT, Gale T, Fulford LG, Reis-Filho JS, Lakhani SR. The diagnosis and management of pre-invasive breast disease: pathology of atypical lobular hyperplasia and lobular carcinoma in situ. Breast Cancer Res 2003; 5 : 258-62.

9. Oppong BA, King TA. Recommendations for women with lobular carcinoma in situ (LCIS). Oncology (Williston Park) 2011; 25: 1051-6, 1058.

10. Polom K, Murawa D, Pawelska A, Murawa P. Atypical lobular hyperplasia and lobular carcinoma in situ without other high-risk lesions diagnosed on vacuum-assisted core needle biopsy. The problem of excisional biopsy. Tumori 2009; 95: 32-5.

11. Flegg KM, Flaherty JJ, Bicknell AM, Jain S. Surgical outcomes of borderline breast lesions detected by needle biopsy in a breast cancer screening program. World J Surg Oncol 2010; 8: 78.

12. Meroni S, Bozzini AC, Pruneri G, et al. Underestimation rate of lobular intraepithelial neoplasia in vacuum-assisted breast biopsy. Eur Radiol 2014; 24: 1651-8.

13. Renshaw AA, Derhagopian RP, Martinez P, Gould EW. Lobular neoplasia in breast core needle biopsy specimens is associated with a low risk of ductal carcinoma in situ or invasive carcinoma on subsequent excision. Am J Clin Pathol 2006; 126: 310-3.

14. Beute BJ, Kalisher L, Hutter LV. Lobular carcinoma in situ of the breast: clinical, pathologic, and mammographic features. AJR Am J Roentgenol 1991; 157: 257-65.

15. Schnitt SJ, Morrow M. Lobular carcinoma in situ: current concepts and controversies. Semin Diagn Pathol 1999; 16: 209-23.

16. Arpino G, Allred DC, Mohsin SK, Weiss HL, Conrow D, Elledge RM. Lobular neoplasia on core-needle biopsy clinical significance. Cancer 2004; 101: 242-50.

17. Scoggins M, Krishnamurthy S, Santiago L, Yang W. Lobular carcinoma in situ of the breast: clinical, radiological, and pathological correlation. Acad Radiol 2013; 20: 463-70.

18. Esserman LE, Lamea L, Tanev S, Poppiti R. Should the extent of lobular neoplasia on core biopsy influence the decision for excision? Breast J 2007; 13: 55-61. 
19. Murray MP, Luedke C, Liberman L, Nehhozina T, Akram M, Brogi E. Classic lobular carcinoma in situ and atypical lobular hyperplasia at percutaneous breast core biopsy. Cancer 2013; 119: 1073-9.

20. Cangiarella J, Guth A, Axelrod D, et al. Is surgical excision necessary for the management of atypical lobular hyperplasia and lobular carcinoma in situ diagnosed on core needle biopsy? A report of 38 cases and review of the literature. Arch Pathol Lab Med 2008; 132: 979-83.

21. Szynglarewicz B, Matkowski R, Halon A, et al. Lobular neoplasia found on breast biopsy: marker of increased risk of malignancy or direct pre-cancerous lesion? Folia Histochem Cytobiol 2011; 49: 417-24.

22. Middleton LP, Grant S, Stephens T, Stelling CB, Sneige N, Sahin AA. Lobular carcinoma in situ diagnosed by core needle biopsy: when should it be excised? Mod Pathol 2003; 16: 120-9.

23. Oliveira TM, Elias J Jr, Melo AF, et al. Evolving concepts in breast lobular neoplasia and invasive lobular carcinoma, and their impact on imaging methods. Insights Imaging 2105; 5: 183-94.

24. Rendi MH, Dintzis SM, Lehman CD, Calhoun KE, Allison KH. Lobular in-situ neoplasia on breast core needle biopsy: imaging indication and pathologic extent can identify which patients require excisional biopsy. Ann Surg Oncol 2012; 19: 914-21.

25. Friedlander LC, Roth SO, Gavenosis SC. Results of MR imaging screening for breast cancer in high-risk patients with lobular carcinoma in situ. Radiology 2011; 261: 421-7.

26. Sung JS, Malak SF, Bajaj P, Alis R, Dershaw DD, Morris EA. Screening breast MR imaging in women with a history of lobular carcinoma in situ. Radiology 2011; 261: 414-20.

27. Port ER, Park A, Borgen PI, Morris E, Montgomery LL. Results of MRI screening for breast cancer in high-risk patients with LCIS and atypical hyperplasia. Ann Surg Oncol 2007; 14: 1051-7.

28. King TA, Muhsen S, Patil S, et al. Is there a role for routine screening MRI in women with LCIS? Breast Cancer Res Treat 2013; 142: 445-53.

29. Schwartz T, Cyr A, Margenthaler J. Screening breast magnetic resonance imaging in women with atypia or lobular carcinoma in situ. J Surg Res 2015; 193: 519-22.

30. Sneige N, Wang J, Baker BA, Krishnamurthy S, Middleton LP. Clinical, histopathologic, and biologic features of pleomorphic lobular (ductal-lobular) carcinoma in situ of the breast: a report of 24 cases. Mod Pathol 2002; 15: 1044-50.

31. Chen YY, Hwang ES, Roy R, et al. Genetic and phenotypic characteristics of pleomorphic lobular carcinoma in situ of the breast. Am J Surg Pathol 2009; 33: 1683-94.

32. Elsheikh TM, Silverman JF. Follow-up surgical excision is indicated when breast core needle biopsies show atypical lobular neoplasia or lobular carcinoma in situ: a correlative study of 33 patients with review of the literature. Am J Surg Pathol 2005; 29: 534-43.

33. Lechner MC, Jackman RJ, Brem RF, Evans WP III, Parker SH, Smid AA. Lobular carcinoma in situ and atypical lobular hyperplasia at percutaneous biopsy with surgical correlation: a multi-institutional study. Radiology 1999; 213: 106

34. Menon S, Porter GJ, Evans AJ, et al. The significance of lobular neoplasia on needle core biopsy of the breast. Virchows Arch 2008; 452: 473-9.

35. Nagi CS, O'Donnell JE, Tismenetsky M, Bleiweiss IJ, Jaffer SM. Lobular neoplasia on core needle biopsy does not require excision. Cancer 2008; 112: 2152-8.
36. Hwang H, Barke LD, Mendelson EB, Susnik B. Atypical lobular hyperplasia and classic lobular carcinoma in situ in core biopsy specimens: routine excision is not necessary. Mod Pathol 2008; 21: 1208-16.

37. Sickles EA. Periodic mammographic follow-up of probably benign lesions: results in 3,184 consecutive cases. Radiology 1991; 179: 463-8.

38. Parkin CK, Garewal S, Waugh P, Maxwell AJ. Outcomes of patients with lobular in situ neoplasia of the breast: the role of vacuum-assisted biopsy. Breast 2014; 23: 651-5. 